\title{
Depression, anxiety and self-esteem in adolescent girls with polycystic ovary syndrome
}

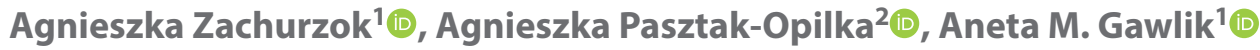 \\ ${ }^{1}$ Department of Pediatrics and Pediatric Endocrinology, Medical University of Silesia, School of Medicine in Katowice, Poland \\ ${ }^{2}$ Institute of Psychology, Faculty of Social Sciences, University of Silesia, Katowice, Poland
}

\begin{abstract}
Objectives: Objective of the study was to evaluate the depression, anxiety and perceived stress level in adolescent girls with diagnosed polycystic ovary syndrome (PCOS), as well as to assess their body and self-esteem and its impact on emotional status.

Material and methods: In 27 adolescent girls with confirmed diagnosis of PCOS (study group) as well as 27 healthy, regularly menstruating, age and BMI matched girls (control group) Hospital Anxiety and Depression Scale (HADS), Perceived Stress Scale-10 (PSS-10), Rosenberg Self-Esteem Scale (RSES) and Body-Esteem Scale (BES) containing three subscales (sexual attractiveness, weight concern, physical condition) were performed.

Results: There were no significant differences between PCOS group and control group in depression and PSS-10 scores, but the anxiety score was significantly higher in control than in PCOS group $(9.6 \pm 3.0$ vs $7.3 \pm 3.9, p=0.02)$. Moreover, in BES subscales' scores there were no significant differences between the groups, whereas RSES score was significantly higher in PCOS group $(25.0 \pm 7.1$ vs $28.3 \pm 4.6, p=0.04)$. In PCOS group anxiety score was related to PSS- 10 score $(r=0.56$, $p=0.005)$. Moreover, we found that obesity was negatively related to anxiety $\left(r_{y}=-0.4, p=0.04\right)$, depression $\left(r_{y}=-0.48\right.$, $p=0.02)$, PSS $-10\left(r_{y}=-0.59, p=0.004\right)$ and physical condition scores $\left(r_{y}=-0.44, p=0.04\right)$. In girls with PCOS the more severe depression the worse weight control $\left(r_{y}=-0.56, p=0.04\right)$.

Conclusions: We conclude that in adolescent girls PCOS is not related to anxiety and depressive symptoms as well as poor self-esteem.
\end{abstract}

Key words: adolescent girls; polycystic ovary syndrome; depression; anxiety; self-esteem

Ginekologia Polska 2021; 92, 6: 399-405

\section{INTRODUCTION}

Polycystic ovary syndrome (PCOS) is one of the most common endocrinopathies in adult women, with characteristic features of anovulation, clinical and biochemical hyperandrogenism. It is a chronic condition leading to the occurrence of many relevant concerns, such as fear of infertility, obesity and its metabolic complications and finally unfeminine, unattractive appearance due to hirsutism, acne or alopecia. PCOS begins in adolescent years, however in adolescent girls, clinical features of the syndrome could be less pronounced or be on the border with adolescent physiology. Nevertheless, in adolescents, quality of life seems to be affected by similar signs and symptoms of PCOS as in adult women: excessive weight, clinical hyperandrogenism, menstrual disturbances and infertility [1].
There are many studies on psychological well-being, emotional problems and stress that arose from clinical features, comorbidities and consequences of PCOS in adult women [2, 3]. Cooney et al. [2] in a meta-analysis showed that women with PCOS have significantly higher risk of moderate and severe depressive and anxiety symptoms, independently of obesity. Moreover, in women with PCOS increased risk of conversion to depression with time is observed [3]. Their perceived stress in higher that in healthy women. The concerns about the emotional status led to recommendation for screening for depression and anxiety in all PCOS women, contained in guidelines of the assessment and management of PCOS published by the Androgen Excess and PCOS Society [4].

\author{
Corresponding author: \\ Agnieszka Zachurzok \\ Department of Pediatrics and Pediatric Endocrinology, Medical University of Silesia, School of Medicine in Katowice, 15 Poniatowskiego St, 40-752 Katowice, Poland \\ e-mail: agnieszkazachurzok@poczta.onet.pl
}


In today's culture physical attractiveness is believed to be one of the main determinants of the success and physical features characteristic for PCOS, such as excessive body weight, hirsutism and acne, are remote from the beauty standards [5]. They can lead to dissatisfaction with the appearance and lower self-esteem. In adult women PCOS is related to greater body dissatisfaction [6]. Moreover Bazarganipour et al., [7] shoved that PCOS women with amenorrhea had lover level of self-esteem and infertility was negatively associated with both self-esteem and body satisfaction. Also in adolescents some researches showed that visible symptoms of androgen excess influence self-esteem and provoke discomfort [8].

The data about the psychological well-being of adolescent girls with PCOS are less numerous, inconsistent and sometimes even conflicting. Considering the duration of the disease, the severity and type of the symptoms, as well as the specificity of lifetime period, the psychological and emotional consequences could be different than in adult patients, associated with different factors. The aim of the study was to evaluate the depression, anxiety and perceived stress level in adolescent girls with diagnosed PCOS, as well as to assess their body and self-esteem and its impact on emotional status.

\section{MATERIAL AND METHODS}

We recruited 54 adolescent girls admitted to the Department of Pediatric Endocrinology due to suspicion of hirsutism and/or menstrual disturbances. Twenty-seven adolescent girls with confirmed diagnosis of PCOS, prior to treatment introduction, were included into the study group and 27 healthy, regularly menstruating, non-hirsute, age and BMI matched girls who voluntarily agreed to participate into the study were included into the control group. PCOS was diagnosed according to the criteria proposed by Ibanez et al [9]. A diagnosis of PCOS was made when both criteria were present: menstrual disturbances (oligomenorrhea, secondary amenorrhea) and clinical or biochemical hyperandrogenism. Oligomenorrhea was defined as menstrual cycles longer than 45 days in the last six months (or less than 6 menstrual cycles during the last year) and secondary amenorrhea as a lack of menstruation in the last three months. Hirsutism, diagnosed if the modified Ferriman-Gallwey score was $\geq 8$, was considered as a sign of clinical hyperandrogenism. Biochemical hyperandrogenism was identified by testosterone level $\geq 55 \mathrm{ng} / \mathrm{mL}$ [10]. The exclusion criteria were other hormonal disturbances (abnormal thyroid function, hyperprolactinaemia, congenital adrenal hyperplasia) and former serious psychiatric disease.

The study was conducted according to Helsinki declaration and approved by the Ethic Committee of The Medical University of Silesia (KNW/0022/KB1/79/12). Informed consent was obtained from each participant and their parent/guardian.

In all participants somatic development data (weight, height) were measured, hirsutism score was assessed by the same observer (AZ). BMI was calculated and obesity was diagnosed in participants with BMI exceeded $97^{\text {th }}$ percentile. Serum level of androstenedione $(A)$, testosterone $(T)$, 17-hydroxyprogesterone (17OHP), dehydroepiandrosterone-sulfate (DHEAS) and estradiol $\left(\mathrm{E}_{2}\right)$ were measured during follicular phase of menstrual cycle (2-5 day of the cycle) or after 3 months from last menstruation. Serum levels of T, DHEAS, and $E_{2}$ were measured using chemiluminescent immunoassay by Immulite 2000 analyzer (DPC, USA). 17OHP and $A$ were measured by enzyme-linked immunosorbent assay (DRG Diagnostics GmbH, Germany).

\section{Psychological assessment}

Hospital Anxiety and Depression Scale (HADS), Perceived Stress Scale-10 (PSS-10), Rosenberg Self-Esteem Scale (RSES) and Body-Esteem Scale (BES) were performed in all patients and controls. The questionnaires were anonymous and independently answered during unlimited time. The average time of completion was about 20 minutes.

HADS is a questionnaire validated for Polish adolescents, consisted with seven questions dedicated to the assessment of anxiety, and seven to assess depression symptoms [11]. Scores of 8 or above are considered abnormal, characteristic for mild (score of 8-10), moderate (score of 11-14) and severe (score above 15) anxiety/depression symptoms.

PSS-10 is a questionnaire consisting of 10 items measuring the perception of stress in the last month, developed by Cohen et al. in 1983 year [12], validated for Polish population by Juczynski and Oginska-Bulik [13]. It measures the degree to which situation in one's life are appraised as stressful.

RSES is diagnostic 10-item tool assessing global self-worth, developed by Rosenberg in 1965 year. It uses four grade Likert scale from strongly agree to strongly disagree [14].

BES is 35-item questionnaire assessing the body image in three subscales: 1 ) sexual attractiveness - related to the body parts those which appearance cannot generally be changed through exercise but only through cosmetics; 2) weight concern - related to body parts that can be physically altered through exercise or control of food intake; 3) physical condition - related to stamina, strength and agility. It was developed by Franzoi and Shields in 1984 year and validated for Polish population by Lipowska and Lipowski $[15,16]$. It uses 5-point Likert scale where 1 means "I have strong negative feelings" and 5 - "I have strong positive feelings".

The statistical analysis was performed using Statistica $12,0 \mathrm{PL}$. The quantitative variables were described with 
mean (SD) and qualitative variables were defined by frequency (\%). To evaluate the normality of variables Shapiro-Wilk test was used. We used $x^{2}$ test for qualitative data comparison of groups, and independent sample t-Student test and U Mann-Whitney test for quantitative data comparison of groups, as appropriate. Correlation analysis was performed using Pearson correlation coefficient for normally distributed samples, Spearman correlation coefficient for non-normally distributed data, and Gamma correlation for non-normally distributed data with many tide ranks. $P$ value $<0.05$ was considered statistically significant. To evaluate variables effecting the strength of the HADS scales, standard multiple regression analysis was performed with $\beta$ coefficient interpretation regarding the strength and direction of the relationship between variables.

\section{RESULTS}

Twenty-seven adolescent girls with PCOS aged 13.6-17.9 years, as well as 27 healthy, regularly menstruated girls aged 14.3-18.0 years were involved into the study. The groups were age and BMI matched so the chronological age, BMI and BMI z-score did not differ significantly between the groups. Clinical and hormonal characteristics are presented in Table 1. The menstrual disturbances were present in all girls from the study group and in all girls from the control group the menstrual cycles were regular. Obesity was seen in nine girls from the study group (33.3\%) and 13 from the control group (48\%) $(p=0.2)$. The biochemical and/or clinical hyperandrogenism were present in all girls

Table 1. Clinical and hormonal characteristics of adolescent girls with polycystic ovary syndrome (PCOS) and control group of healthy girls

\begin{tabular}{|c|c|c|c|}
\hline & $\begin{array}{l}\text { Girls with } \\
\text { PCOS }(n=27)\end{array}$ & $\begin{array}{l}\text { Control } \\
\text { group }(n=27)\end{array}$ & $\mathbf{p}$ \\
\hline $\begin{array}{l}\text { Chronological age } \\
\text { [years] }\end{array}$ & $16.7 \pm 1.2$ & $16.1 \pm 1.1$ & NS \\
\hline $\begin{array}{l}\text { Gynaecological age } \\
\text { [months] }\end{array}$ & $52.5 \pm 19.4$ & $40.7 \pm 16.9$ & NS \\
\hline Cycle duration [days] & $108.6 \pm 60.8$ & $29.7 \pm 4.8$ & $<0.001$ \\
\hline BMI z-score & $1.1 \pm 0.9$ & $1.0 \pm 1.0$ & NS \\
\hline $\begin{array}{l}\text { Ferriman-Gallwey } \\
\text { score }\end{array}$ & $5.2 \pm 4.4$ & $3.3 \pm 2.9$ & NS \\
\hline Testosterone [ng/dL] & $61.2 \pm 23.2$ & $45.0 \pm 14.2$ & 0.009 \\
\hline $\begin{array}{l}\text { Androstenedione } \\
{[\mathrm{ng} / \mathrm{mL}]}\end{array}$ & $4.6 \pm 1.3$ & $3.3 \pm 0.8$ & $<0.001$ \\
\hline DHEAS $[\mu \mathrm{g} / \mathrm{dL}]$ & $312.7 \pm 134.5$ & $266.2 \pm 88.0$ & NS \\
\hline $\begin{array}{l}\text { 170Hprogesteron } \\
{[\mathrm{ng} / \mathrm{mL}]}\end{array}$ & $2.4 \pm 1.5$ & $2.4 \pm 1.8$ & NS \\
\hline Estradiol [pmol/L] & $155.0 \pm 56.0$ & $107.0 \pm 80.4$ & 0.052 \\
\hline
\end{tabular}

PCOS - polycystic ovary syndrome; BMI — body mass index; DHEAS — dehydroepiandrosterone-sulfate; NS — nonsignificant from the study group. Hirsutism was present in 10 subjects with PCOS (38.5\%). However, most of the girls presented mild hirsutism (8-15 points in Ferriman-Gallwey scale) and only in one girl the excessive hair growth was assessed at 16 points. Biochemical hyperandrogenism was found significantly more often in girls from the study group than from the control group [ 15 girls ( $57.7 \%)$ vs 5 girls (23.8\%), $p=0.02$ ].

There were no significant differences in the depression score and PSS-10 score between the groups ( $p>0.05$ ) (Tab. 2). But anxiety score was significantly higher in the control group than in study group $(p=0.02)$. Moderate and severe anxiety was found in five (18.5\%) girls with PCOS and in $14(51.8 \%)$ girls from the control group $(p=0.01)$, whereas moderate and high depression was present in one girl from the study group (3.7\%) and in two girls from the control group (7.2\%) ( $p>0.05)$ (Fig. 1). There were no significant differences between PCOS and the control group for all subscales of BES. Whereas RSES score was significantly higher in PCOS than in the control group $(p=0.04)$.

In the study group we found significant positive correlation between anxiety score and PSS-10 score $(r=0.56$, $p=0.005)$ and negative with RSES score $(r=-0.44, p=0.03)$. PSS- 10 score correlated negatively with RSES score $(r=-0.58$, $p=0.003)$. Surprisingly, depression score and PSS- 10 score correlated negatively with BMI $(r=-0.38, p=0.049 ; r=-0.43$, $p=0.03$, respectively), whereas RSES score correlated positively with BMI ( $r=0.53, p=0.004)$. In the study group obesity was negatively related to anxiety score $\left(r_{y}=-0.4\right.$, $p=0.04)$, depression score $\left(r_{y}=-0.48, p=0.02\right)$ and PSS-10 score $\left(r_{y}=-0.59, p=0.004\right)$ as well as physical condition score $\left(r_{y}=-0.44, p=0.04\right)$. Moreover, we found that the more severe depression, the worse weight control $\left(r_{y}=-0.56\right.$,

Table 2. Psychological assessment of adolescent girls with polycystic ovary syndrome (PCOS) and control group of healthy girls

\begin{tabular}{|c|c|c|c|}
\hline & $\begin{array}{l}\text { Girls with } \\
\text { PCOS }(n=27)\end{array}$ & $\begin{array}{l}\text { Control group } \\
(n=27)\end{array}$ & $\mathbf{p}$ \\
\hline \multicolumn{4}{|c|}{ Hospital Anxiety and Depression Scale } \\
\hline anxiety [scores] & $7.3 \pm 3.9$ & $9.6 \pm 3.0$ & 0.02 \\
\hline depression [scores] & $4.2 \pm 2.9$ & $5.1 \pm 3.4$ & NS \\
\hline $\begin{array}{l}\text { Perceived Stress Scale } \\
-10 \text { [scores] }\end{array}$ & $22.0 \pm 3.4$ & $22.7 \pm 3.3$ & NS \\
\hline $\begin{array}{l}\text { Rosenberg Self-Esteem } \\
\text { Scale [scores] }\end{array}$ & $28.3 \pm 4.6$ & $25.0 \pm 7.1$ & 0.04 \\
\hline \multicolumn{4}{|l|}{ Body Self Esteem } \\
\hline $\begin{array}{l}\text { sexual attractiveness } \\
\text { [score] }\end{array}$ & $44.3 \pm 5.2$ & $44.0 \pm 9.6$ & NS \\
\hline weight concern [score] & $28.1 \pm 8.0$ & $25.3 \pm 10.5$ & NS \\
\hline physical condition [scores] & $30.7 \pm 5.2$ & $30.0 \pm 6.5$ & NS \\
\hline
\end{tabular}

PCOS - polycystic ovary syndrome; NS - nonsignificant 
HADS anxiety

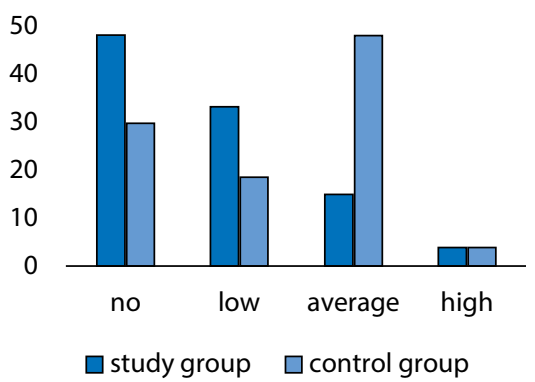

HADS depression

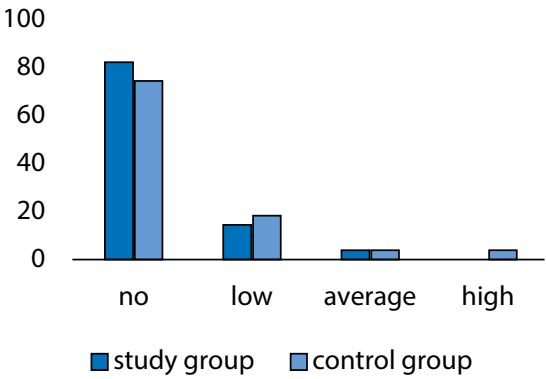

Figure 1. Distribution of no, low, average and high anxiety and depression symptoms in the study and control groups; Scores below 8 is characteristic for no anxiety/depression symptoms, 8-10 scores for mild, 11-14 score for moderate and > 15 score for severe anxiety/depression symptoms; HADS - Hospital Anxiety and Depression Scale

$\mathrm{p}=0.04)$. We found no significant relationship between depression, anxiety, PSS-10, RSES and BES subscales scores and clinical and biochemical hyperandrogenism, as well as severity of clinical phenotype of PCOS.

In multiple regression analysis, PSS-10, RSES, sexual attractiveness, weight concern, physical condition scores, $\mathrm{BMI}$, hirsutism scores were included in the analysis as scale variables. The anxiety score was significantly associated with the PSS-10 score $(\beta=0.33, p=0.05)$ in the control group but not in the study group. In examining depression score we found significant association with weight concern score in the control group $(\beta=0.72, p=0.01)$ but not in the study group.

\section{DISCUSSION}

The study was designed to estimate the depression and anxiety occurrence in adolescent girls with PCOS as well as to assess their self-esteem. We tried to study the relationship of depression, anxiety and self-esteem with clinical and biochemical features of hyperandrogenism. The anxiety score was higher in control than in PCOS group, but we did not find any differences between the groups in depression and PSS10 scores. Researches showed that there is significant relationship between depression and anxiety and PCOS in adult women $[17,18]$. The prevalence of depression and anxiety symptoms in women with PCOS is $29-50 \%$ and $57 \%$ respectively $[19,20]$. Moreover, the symptoms are related to hyperandrogenism, insulin resistance and in some research to obesity [20]. However, such relationships are not so obvious in adolescent girls with PCOS. The studies on psychological well-being in PCOS girls are on many fields scarce and conflicting. Quality of life in adolescent girls with PCOS by some researchers is assessed as negatively influenced by the syndrome [21], whereas in other studies no relationship was found [22]. Ghazeeni et al. [23], showed no significant difference with anxiety and depression status between the adolescent girls with PCOS and healthy age-matched girls. On the other hand, Eneksis et al. [24], found higher level of anxiety and depression in PCOS than in controls. However, their study group was older than our PCOS group, so the persistence of the clinical symptoms could last longer. And as showed Guidi et al. [25], significantly higher anxiety and depression, impaired well-being and quality of life was found among adolescents with hirsutism. However, in their research the hirsutism score was self-estimated, meaning strongly subjective assessment. Neither menstrual irregularity, nor PCOS was associated with higher psychological distress. They concluded that among late adolescents and young women more important is concern about physical appearance then reproductive problems [25]. In our study we did not find the difference in depression score between the groups, whereas anxiety score was significantly higher in control group than in study group, significantly more girls from control group experienced moderate and severe anxiety. There could be several reasons underlying this lack of relationship. First, some symptoms of PCOS occurring during adolescence overlap findings that are considered physiological, normal during puberty [26]. Irregular menses occurring in many girls especially in the first years after menarche should not be treated as symptoms of PCOS, as well acne, which is typical physiological finding during puberty. So, these features could be perceived by adolescent girls as not pathological. Also, the main clinical presentation of hyperandrogenism, hirsutism could be mild in adolescent years, increases its severity with age. The severity of hirsutism in studied girls with PCOS was also not very high, only in one girl from study group her excessive hair growth was assessed at more than 15 score in Ferriman-Gallwey scale. Most of the girls from the study group have mild hirsutism, which could be not very relevant to emotional distress.

The reason for lack of anxiety and depression symptoms in our study group could be short duration of the symptoms, and there is also the suggestion that the predictor of the depression in women with PCOS is the age [26]. It is 
postulated that not clinical hyperandrogenism per se but the chronic character of clinical features of PCOS could be associated with increased risk of depression and anxiety disturbances. In adult women the symptoms of menstrual irregularities and clinical hyperandrogenism last for many years with unsatisfactory results of treatment, leading to increase in emotional and psychological distress. And finally, very important concern which arises from the age and period of life of young adult woman is infertility.

The lack of difference between depression score with higher anxiety in control group could be also related to the control group characteristic. We involved into the control group adolescents with regular cycles without clinical hyperandrogenism, however they were referred to our clinic for evaluation due to a suspicion of hormonal disorders. All endocrine diseases were excluded in this group of girls however it seems that not real, objective physical signs of endocrine disturbances are important for the level of depression or anxiety but the subjective perception of the complains. Similar relationship we saw in our study group in respect to BMI. BMI correlated negatively with depression score and PSS-10 and positively with RSES, whereas more severe depression was related to the worse weight control. So, the issue is not a real weight status but how it is perceived. The similar depression score and lower anxiety could be related to the social and medical support that PCOS subjects get from the family and professionals as well from the fact that the cause of their symptoms was discovered. In the control group, with strong subjective beliefs of hormonal disturbances, the higher anxiety can be related to lack of symptoms interpretation despite of staying in hospital. The regression analysis, showing in the control group significant influence of stress on anxiety score as well as weight concern on depression score, seems to confirm these arguments.

Body image is a mental picture of one's body and it is highly subjective [7]. Usually, low self-esteem is associated with anxiety, depression, whereas high self-esteem may serve as a protection in coping with new or chronic disease [7]. In our study we also found significant negative relationship between anxiety and PSS-10 score and RSES score. However, the results about relationship of hyperandrogenism with self-esteem are conflicting [27]. According to Bazarganipour et al. [7] adult women with PCOS have poorer self-esteem and self-image satisfaction because of clinical symptoms as menstrual disorders, obesity and clinical hyperandrogenism (acne, alopecia, hirsutism). They found that women with PCOS with menstrual disorders had the greater body dissatisfaction, linking it especially with the fertility concern [7]. However, they examined women from Iran, where strong social pressure to have a child soon after marriage is present. In Poland, like in other European countries, there is a tendency to postpone the maternity. Accord- ing to Eurostat, in recent years the average age of a Polish woman at the birth of the first child is 27.3 years [28]. So, the concern about fertility is also postpone in time. Keegan et al. [27] did not report the difference in self-esteem between women with and without hirsutism. In adolescents with PCOS, Coban et al. [22] showed no significant difference compared with control group. They also did not find any relationship between RSES score and clinical symptoms of hyperandrogenism [22]. In our study RSES score was even higher in PCOS group than in control one. Drosdzol et al. [29] showed that in adolescents with hirsutism RSES score was similar as in healthy peers. These results refer to RSES, which is very reliable but general tool that does not measure items of self-esteem related to physical appearance. That is why we decided to use also BES to assess body self-esteem in the three areas: sexual attractiveness, weight concern and physical condition. And we found also lack of significant differences in all three BES subscales scores between the groups, leading to the conclusion that the body self-esteem related to sexual attractiveness, weight concern and physical condition is not influenced by PCOS in adolescent girls. To the similar conclusion arrived also Annagur et al., [30], finding that higher body satisfaction was present in PCOS women than in healthy control.

Previous research suggested that excessive body weight gain during adolescence may be related to the depression, negative mood state and poor self-esteem [31]. Surprisingly, we found negative relationship between BMI and obesity and depression, anxiety and PSS-10 scores, meaning that the higher weight the lower level of depression, anxiety and perceived stress. It contrasts with other research in adults and adolescents [17, 18]. Moreover, RSES score significantly correlated with BMI. However, in our previous research we showed that in adolescent girls with clinical hyperandrogenism and obesity, BMI z-score was positively related to social competences [32]. And the group with strongest inclinations to withdrawing from social relations was the non-hirsute, non-obese subgroup. It could be due to that excessive body weight is no longer the feature that stigmatize anybody, especially if the whole family is obese or overweight and increasing amounts of peers have excessive body weight. Possible explanation is also the subjective, positive self-assessment of own body image which could neutralize negative self-assessment [33]. Obesity, lasting from early childhood could lead also to the feeling of "getting used" to this image of the body, does not influence body self-esteem. The data about the influence of obesity on psychological distress are no so clear. In a meta-analysis, Rankin et al., [31] tried to clarify the relationship between excessive weight in children and depression and anxiety. They found that depression could be related to obesity in childhood, whereas the data about relation to anxiety disor- 
ders are not so obvious. However, study findings analyzed in this meta-analysis vary - in couple of them, obese children were found to be significantly more likely to experience depression. In other studies depression risk was only modestly greater than in general population or the association was weak [31]. Moreover, three meta-analysis focusing on emotional distress and mental disorder in PCOS women suggested that BMI and hirsutism have a small or moderate effect on anxiety and depression [2, 34, 35].

\section{CONCLUSIONS}

We conclude that in adolescent girls PCOS is not related to anxiety and depressive symptoms as well as poor self-esteem. However, considering the possible relationship between PCOS and emotional distress in adult women, due to long lasting, chronic medical condition, patients with PCOS should be monitored for emotional and psychological problems from adolescence to adulthood.

The study limitation is a relatively small number of recruited patients. Increasing the size of the study group might demonstrate more reliable estimates for several independent variables as well determine the ability to detect further subtle relationships.

\section{Funding}

The research was supported by grant of Medical University of Silesia KNW 1-113/N/3/0.

\section{Conflict of interest}

The authors declare no conflict of interest.

\section{REFERENCES}

1. Kaczmarek C, Haller DM, Yaron M. Health-Related Quality of Life in Adolescents and Young Adults with Polycystic Ovary Syndrome: A Systematic Review. J Pediatr Adolesc Gynecol. 2016; 29(6): 551-557, doi: 10.1016/j. jpag.2016.05.006, indexed in Pubmed: 27262833.

2. Cooney LG, Lee I, Sammel MD, et al. High prevalence of moderate and severe depressive and anxiety symptoms in polycystic ovary syndrome: a systematic review and meta-analysis. Hum Reprod. 2017; 32(5): 1075-1091, doi: 10.1093/humrep/dex044, indexed in Pubmed: 28333286.

3. Rowlands IJ, Teede H, Lucke J, et al. Young women's psychological distress after a diagnosis of polycystic ovary syndrome or endometriosis. Hum Reprod. 2016; 31(9): 2072-2081, doi: 10.1093/humrep/dew174, indexed in Pubmed: 27412249.

4. Teede HJ, Misso ML, Costello MF, et al. International PCOS Network. Recommendations from the international evidence-based guideline for the assessment and management of polycystic ovary syndrome. Fertil Steril. 2018; 110(3):364-379, doi: 10.1016/j.fertnstert.2018.05.004, indexed in Pubmed: 30033227.

5. Zachurzok A, Pasztak-Opilka A, Forys-Dworniczak E, et al. Are Psychosocial Consequences of Obesity and Hyperandrogenism Present in Adolescent Girls with Polycystic Ovary Syndrome? Int J Endocrinol. 2018; 2018: 3269618, doi: 10.1155/2018/3269618, indexed in Pubmed: 30147723.

6. Himelein MJ, Thatcher SS. Polycystic ovary syndrome and mental health: A review. Obstet Gynecol Surv. 2006; 61(11): 723-732, doi: 10.1097/01. ogx.0000243772.33357.84, indexed in Pubmed: 17044949.

7. Bazarganipour F, Ziaei S, Montazeri A, et al. Body image satisfaction and self-esteem status among the patients with polycystic ovary syndrome. Iran J Reprod Med. 2013; 11(10): 829-836.
8. Jones $\mathrm{GL}$, Hall JM, Lashen $\mathrm{HL}$, et al. Health-related quality of life among adolescents with polycystic ovary syndrome. J Obstet Gynecol Neonatal Nurs. 2011 ; 40(5): 577-588, doi: 10.1111/j.1552-6909.2011.01279.x, indexed in Pubmed: 22273414.

9. Ibáñez L, Oberfield SE, Witchel S, et al. An International Consortium Update: Pathophysiology, Diagnosis, and Treatment of Polycystic Ovarian Syndrome in Adolescence. Horm Res Paediatr. 2017; 88(6): 371-395, doi: 10.1159/000479371, indexed in Pubmed: 29156452.

10. Carmina E, Oberfield SE, Lobo RA. The diagnosis of polycystic ovary syndrome in adolescents. Am J Obstet Gynecol. 2010; 203(3): 201.e1-201. e5, doi: 10.1016/j.ajog.2010.03.008, indexed in Pubmed: 20435290.

11. Mihalca AM, Pilecka W. The factorial structure and validity of the Hospital Anxiety and Depression Scale (HADS) in Polish adolescents. Psychiatr Pol. 2015; 49(5): 1071-1088, doi: 10.12740/PP/38139, indexed in Pubmed: 26688854.

12. Cohen S, Kamarck T, Mermelstein R. A Global Measure of Perceived Stress. Journal of Health and Social Behavior. 1983; 24(4): 385, doi: $10.2307 / 2136404$

13. Juczynski Z, Oginska-Bulik N. Methods to measure stress and coping. Pracownia Testów Psychologicznych, Warszawa 2009.

14. Rosenberg M. Society and the Adolescent Self-Image. 1965, doi: $10.1515 / 9781400876136$.

15. Franzoi SL, Shields SA. The Body Esteem Scale: multidimensional structure and sex differences in a college population. J Pers Assess. 1984; 48(2): 173-178, doi: 10.1207/s15327752jpa4802 12, indexed in Pubmed: 6726603.

16. Lipowska $M$, Lipowski M. Original article Polish normalization of the Body Esteem Scale. Health Psychology Report. 2013; 1: 72-81, doi: 10.5114/hpr.2013.40471.

17. Adali E, Yildizhan R, Kurdoglu $M$, et al. The relationship between clinico-biochemical characteristics and psychiatric distress in young women with polycystic ovary syndrome. J Int Med Res. 2008; 36(6): 1188-1196, doi: 10.1177/147323000803600604, indexed in Pubmed: 19094426.

18. Moran L, Gibson-Helm M, Teede H, et al. Polycystic ovary syndrome: a biopsychosocial understanding in young women to improve knowledge and treatment options. J Psychosom Obstet Gynaecol. 2010; 31(1): 2431, doi: 10.3109/01674820903477593, indexed in Pubmed: 20050767.

19. Deeks AA, Gibson-Helm ME, Teede HJ. Anxiety and depression in polycystic ovary syndrome: a comprehensive investigation. Fertil Steril. 2010; 93(7): 2421-2423, doi: 10.1016/j.fertnstert.2009.09.018, indexed in Pubmed: 20117778.

20. Hollinrake $E$, Abreu A, Maifeld $M$, et al. Increased risk of depressive disorders in women with polycystic ovary syndrome. Fertil Steril. 2007 87(6): 1369-1376, doi: 10.1016/j.fertnstert.2006.11.039, indexed in Pubmed: 17397839 .

21. Trent ME, Rich $M$, Austin SB, et al. Quality of life in adolescent girls with polycystic ovary syndrome. Arch Pediatr Adolesc Med. 2002; 156(6): $556-$ 560, doi: 10.1001/archpedi.156.6.556, indexed in Pubmed: 12038887.

22. Çoban ÖG, Tulacı ÖD, Adanır AS, et al. Psychiatric Disorders, Self-Esteem, and Quality of Life in Adolescents with Polycystic Ovary Syndrome. J Pediatr Adolesc Gynecol. 2019; 32(6): 600-604, doi: 10.1016/j. jpag.2019.07.008, indexed in Pubmed: 31362114.

23. Ghazeeri G, Fakih A, Abbas HA, et al. Anxiety, cognitive, and depressive assessment in adolescents with polycystic ovarian syndrome: a pilot study. J Pediatr Adolesc Gynecol. 2013; 26(5): 269-273, doi: 10.1016/j. jpag.2013.04.005, indexed in Pubmed: 23849090.

24. Emeksiz HC, Bideci A, Nalbantoğlu B, et al. Anxiety and depression states of adolescents with polycystic ovary syndrome. Turk J Med Sci. 2018; 48(3): 531-536, doi: 10.3906/sag-1708-131, indexed in Pubmed: 29914248.

25. Guidi J, Gambineri A, Zanotti L, et al. Psychological aspects of hyperandrogenic states in late adolescent and young women. Clin Endocrinol (Oxf). 2015; 83(6): 872-878, doi: 10.1111/cen.12783, indexed in Pubmed: 25823959.

26. Rahebi SM, Ghanbari A, Reza So, et al. Depression in women with polycystic ovary syndrome: the role of body mass index and infertility on it. Nurs Pract Today. ; 2(4): 152-157.

27. Keegan A, Liao LM, Boyle M.,Hirsutism': a psychological analysis. J Health Psychol. 2003; 8(3): 327-345, doi: 10.1177/13591053030083004, indexed in Pubmed: 14670212

28. https://ec.europa.eu/eurostat/statistics-explained/index.php/Fertility_statistics\#Total_fertility_rate_and_age_of_women_at_birth_of_ first_child. 
29. Drosdzol A, Skrzypulec V, Plinta R. Quality of life, mental health and self-esteem in hirsute adolescent females. J Psychosom Obstet Gynaecol. 2010; 31(3): 168-175, doi: 10.3109/0167482X.2010.501398, indexed in Pubmed: 20626242.

30. Annagür BB, Tazegül A, Akbaba N. Body Image, Self-Esteem and Depressive Symptomatology in Women with Polycystic Ovary Syndrome. Noro Psikiyatr Ars. 2014; 51(2): 129-132, doi: 10.4274/npa.y6778, indexed in Pubmed: 28360612

31. Rankin J, Matthews L, Cobley S, et al. Psychological consequences of childhood obesity: psychiatric comorbidity and prevention. Adolesc Health Med Ther. 2016; 7:125-146, doi: 10.2147/AHMT.S101631, indexed in Pubmed: 27881930.

32. Zachurzok A, Pasztak-Opilka A, Forys-Dworniczak E, et al. Are Psychosocial Consequences of Obesity and Hyperandrogenism Present in
Adolescent Girls with Polycystic Ovary Syndrome? Int J Endocrinol. 2018; 2018: 3269618, doi: 10.1155/2018/3269618, indexed in Pubmed: 30147723.

33. Barker ET, Galambos NL. Body dissatisfaction of adolescent girls and boys: Risk and resource factors. The Journal of Early Adolescence. 2003; 23(2): 141-165.

34. Veltman-Verhulst SM, Boivin J, Eijkemans MJC, et al. Emotional distress is a common risk in women with polycystic ovary syndrome: a systematic review and meta-analysis of 28 studies. Hum Reprod Update. 2012; 18(6): 638-651, doi: 10.1093/humupd/dms029, indexed in Pubmed: 22824735.

35. Barry JA, Kuczmierczyk AR, Hardiman PJ. Anxiety and depression in polycystic ovary syndrome: a systematic review and meta-analysis. Hum Reprod. 2011;26(9): 2442-2451, doi: 10.1093/humrep/der197, indexed in Pubmed: 21725075. 